\title{
BMJ Open Transmuscular quadratus lumborum block versus oblique subcostal transversus abdominis plane block for analgesia in laparoscopic hysterectomy: a randomised single-blind trial
}

\author{
Lvdan Huang, ${ }^{1}$ Liangyu Zheng, ${ }^{1,2}$ Jingxiong Zhang, ${ }^{1}$ Xiaona Zhu, ${ }^{1}$ Linmin Pan, ${ }^{1}$ \\ Yujian Zhang, ${ }^{1}$ Quanguang Wang, ${ }^{1}$ Kejian Shi (D) ${ }^{1}$
}

To cite: Huang $L$, Zheng $L$, Zhang J, et al. Transmuscular quadratus lumborum block versus oblique subcostal transversus abdominis plane block for analgesia in laparoscopic hysterectomy: a randomised singleblind trial. BMJ Open 2021;11:e043883. doi:10.1136/ bmjopen-2020-043883

- Prepublication history for this paper is available online. To view these files, please visit the journal online (http://dx.doi org/10.1136/bmjopen-2020043883).

LH and LZ are joint first authors.

Received 18 August 2020 Accepted 28 July 2021

\section{Check for updates}

(c) Author(s) (or their employer(s)) 2021. Re-use permitted under CC BY-NC. No commercial re-use. See rights and permissions. Published by BMJ.

${ }^{1}$ Anesthesiology, The First Affiliated Hospital of Wenzhou Medical University, Wenzhou, China

${ }^{2}$ Anesthesiology, Zhejiang University School of Medicine Sir Run Run Shaw Hospital, Hangzhou, Zhejiang, China

Correspondence to

Dr Kejian Shi;

wzshikejian@163.com

\section{ABSTRACT}

Objective The transmuscular quadratus lumborum (TQL) block and the oblique subcostal transversus abdominis plane (OSTAP) block both contribute to multimodal analgesia after laparoscopic surgery. The objective of this study was to compare the analgesic effects of the TQL block versus OSTAP block after laparoscopic hysterectomy. Design Prospective single-centre randomised single-blind trial.

Setting University-affiliated hospital.

Participants Patients aged between 18 and 65 years scheduled for laparoscopic hysterectomy.

Interventions Patients were randomised into two groups (1:1 ratio) and received bilateral TQL block or bilateral OSTAP block with $0.375 \%$ ropivacaine $20 \mathrm{~mL}$ on each side before surgery.

Primary and secondary outcome measures The primary outcome measure was the cumulative morphine dose in the first 24 hours. The secondary outcome measures were the morphine consumption at each time interval after surgery, the time from the end of surgery to the first need for morphine, the Numerical Rating Scale (NRS) scores for visceral and incisional pain intensity, and the incidence of adverse events.

Results The cumulative morphine dose was significantly lower in the TQL group than in the OSTAP group (17.2 (12.5) vs 26.1 (13.3) $\mathrm{mg}, \mathrm{p}=0.010$ ). Compared with the OSTAP group, the morphine doses from 6 to 12,12 to 18 , and 18 to 24 hours were significantly lower, the time of first need for morphine was significantly longer and the NRS scores for visceral pain intensity were significantly lower in the TQL group.

Conclusion Compared with the OSTAP block, the TQL block reduced morphine consumption and provided better visceral pain relief with a longer duration of effect after laparoscopic hysterectomy.

Trial registration number Chinese Clinical Trial Registry (ChiCTR1800017995); pre-results.

\section{INTRODUCTION}

Laparoscopic techniques are commonly used in gynaecological surgery. Because gynaecological laparoscopy reduces the pain of
Strengths and limitations of this study

- This is the first randomised controlled trial to evaluate the analgesic effects of the transmuscular quadratus lumborum block versus the oblique subcostal transversus abdominis plane block after laparoscopic hysterectomy.

- This trial evaluated visceral pain in patients following laparoscopic hysterectomy.

- The local anaesthetic was injected under ultrasound guidance, to ensure the process safety and the analgesic effects.

- Patients were not feasible to blind the group allocation due to their different allocated treatments.

- This is a single-centre clinical trial design which may limit the generalisability of the conclusions.

incision compared with laparotomy, visceral pain has become the main postoperative complaint. ${ }^{12}$ In recent years, regional anaesthesia has been increasingly used as a component of multimodal analgesia following the laparoscopic surgery. ${ }^{34}$

Hebbard et a $\tilde{e}$ described oblique abdominal transversus abdominis plane (OSTAP) block in 2010, which can provide effective analgesia for laparoscopic surgery. ${ }^{6}$ However, considering that OSTAP block only blocks the intercostal nerve and its lateral/anterior branches, visceral pain has not been relieved.

The quadratus lumborum (QL) block is increasingly used in laparoscopic surgery in recent years. ${ }^{8-11}$ The QL block causes the local anaesthetic to spread through the thoracolumbar fascia to the thoracic paravertebral space, which may have analgesic effect on incision pain and internal organs. ${ }^{12} 13$ Generally speaking, there are four types of QL block, which are called lateral QL block, 
posterior QL block, transmuscular QL (TQL) block and intramuscular QL block. ${ }^{14}$

There has been no study comparing the TQL block with OSTAP block after laparoscopic hysterectomy. We proposed the hypothesis that the TQL block reduces the analgesic consumption after laparoscopic hysterectomy and provides a better pain relief, compared with the OSTAP block. The primary endpoint was the cumulative morphine dose in the first 24 hours after surgery. Secondary endpoints included the morphine dose from 0 to 1,1 to 6,6 to 12,12 to 18 , and 18 to 24 hours after surgery, the time from the end of surgery to the first need for morphine, the Numerical Rating Scale (NRS) scores for postoperative visceral and incisional pain intensity (at rest and on movement), and the incidence of adverse events.

\section{METHODS}

The trial was registered prior to patient enrolment at the Chinese Clinical Trial Registry (date of registration: 25 August 2018). Written informed consent was obtained from all study participants.

Sixty patients scheduled for laparoscopic hysterectomy were included in this prospective, single-centre randomised trial. Inclusion criteria included age between 18 and 65 years, American Society of Anesthesiologists (ASA) physical status scores of I or II, and body mass index (BMI) between 18 and $28 \mathrm{~kg} / \mathrm{m}^{2}$. Exclusion criteria included a history of allergy of ropivacaine or other drugs used in this trial, a history of alcohol or analgesic dependence, peripheral neuropathy (such as diabetic neuropathy), coagulopathy, skin pathology at the puncture site, a history of severe vomiting or motion sickness, difficulty with communication or unwillingness to receive regional anaesthesia.

Patients were randomised into the TQL and OSTAP groups $(n=30)$ according to a random number table that was generated by SPSS V.22.0 statistical software (IBM Corporation). The trial was a single-blind design. Patients were not feasible to blind the group allocation due to their different allocated treatments. The investigators responsible for data collection and analysis were unaware of group allocation and did not participate in the intervention during the trial.

On the day before surgery, patients were educated to distinguish the postoperative pain in the lower abdomen including incisional versus visceral pain (incisional pain was described as superficial pain localised in the abdominal wall; visceral pain was described as deep abdominal pain that is difficult to localise and is mostly dull, aching or colic pain). Patients were also educated on the NRS pain intensity score $(0=$ no pain, $10=$ worst imaginable pain) and on the use of patient-controlled analgesia (PCA) for postoperative analgesia.

Prior to surgery, all patients were fasted for 8-12 hours in accordance with non-peros (NPO) guidelines. No preoperative medications were given. Intravenous access was established and routine monitors (non-invasive blood pressure, continuous ECG and pulse oximetry) were placed in the operating room.

The regional anaesthesia was performed in the operating room prior to induction of anaesthesia. An ultrasound-guided TQL block or OSTAP block was performed by the same attending anaesthesiologist, who did not participate in data collection or analysis. We used cold stimulus to evaluate the spread of block approximately 30 min after block; and range from T10 to T12 was defined as a successful blockade, which reached the incision plane.

In the TQL group, bilateral TQL block at L2 level was performed with $20 \mathrm{~mL}$ of $0.375 \%$ ropivacaine (LBKL; AstraZeneca AB, Sweden) on each side. The patients were placed in a lateral decubitus position with the operator facing the back. The spinous process line of lumbar vertebra was marked. The 12 th rib was found by using a low-frequency $(2-5 \mathrm{MHz})$ convex probe (SonoSite X-Porte; SonoSite, Bothell, Washington, USA) in the sagittal section. Then the probe was moved medially to check the transverse process of T12 and caudally to check the transverse process of L1 and L2. A vertical line was drawn through the L2 transverse process to the spinous process line. The convex probe scanned along this line, avoiding the costal margin that might be encountered, to find the Shamrock sign: where the psoas muscle was anterior, the spinae muscle was posterior to the transverse process, and the QL muscle was attached to the apex of the transverse process. Using an in-plane technique, a 22G needle (B.Braun Melsungen, Melsungen, Germany) was inserted into the skin in an anteromedial direction through the QL and directed towards the space between the QL and psoas major muscles, determined by an injection of 1-2 mL saline. Then local anaesthetic was injected into the fascial plane (figure 1A).

In the OSTAP group, bilateral OSTAP block was performed with $20 \mathrm{~mL}$ of $0.375 \%$ ropivacaine on each side, as previously described by Chen et al. ${ }^{15}$ Patients were placed in a supine position. A high-frequency (6-12 MHz) linear probe was placed near the xiphoid process, parallel to the costal margin, to identify the rectus abdominis and the underlying transversus abdominis. Using an in-plane technique, a $22 \mathrm{G}$ needle $1-2 \mathrm{~cm}$ medial to the probe was inserted into transversus abdominis plane (TAP) through the rectus muscle. The location of needle tip was confirmed by injection of $1-2 \mathrm{~mL}$ saline. This sequence was repeated four to five times along the oblique subcostal line until the edge of the transducer reached the anterior part of the iliac crest. Then local anaesthetic was evenly injected along the oblique subcostal line (figure 1B).

Patients were placed under general anaesthesia by an anaesthesiologist who was not an investigator for this study and who was unaware of group allocation. Sufentanil $(0.3 \mu \mathrm{g} / \mathrm{kg})$, propofol $(1-2.5 \mathrm{mg} / \mathrm{kg})$ and rocuronium $(0.6 \mathrm{mg} / \mathrm{kg})$ were used for induction. The tidal volume was adjusted to $6-8 \mathrm{~mL} / \mathrm{kg}$ and the respiratory rate to 12-16 breaths/min after tracheal intubation to maintain 

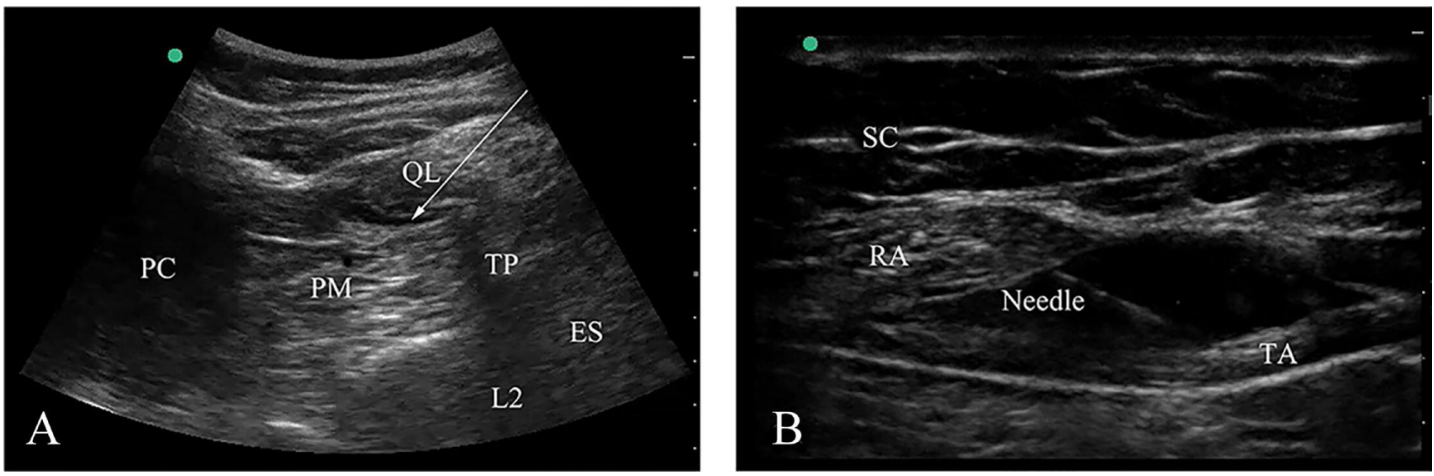

Figure 1 Probe position and sonogram. (A) Ultrasonogram of transmuscular quadratus lumborum (QL) block; (B) ultrasonogram of oblique subcostal transversus abdominis plane block. ES, erector spinae; PC, peritoneal cavity; PM, psoas major; RA, rectus abdominis; SC, subcutaneous; TA, transversus abdominis; TP, transverse process.

end-tidal carbon dioxide pressure between 30 and $45 \mathrm{~mm}$ $\mathrm{Hg}$. Anaesthesia was maintained with propofol $(4-8 \mathrm{mg} /$ $\mathrm{kg} / \mathrm{hour})$, remifentanil $(0.1-0.3 \mu \mathrm{g} / \mathrm{kg} / \mathrm{min})$ and sevoflurane $(1 \%-1.5 \%$ in oxygen). The bispectral index value was maintained between 40 and 60 . Intermittent rocuronium was given when necessary. Ondansetron $(8 \mathrm{mg})$ and flurbiprofen $(100 \mathrm{mg})$ were intravenously administered about $30 \mathrm{~min}$ before the end of the surgery. In addition, intravenous morphine infusion was started in the postanaesthesia care unit with a PCA pump (ZZB-I automatic injection pump driver; Nantong Aipu Medical Instrument Co, Nantong, China) containing $50 \mathrm{mg}$ morphine diluted with saline to a volume of $100 \mathrm{~mL}$. The PCA was programmed to deliver a bolus dose of $1.5 \mathrm{mg}$ morphine, without background infusion, with a lockout of $5 \mathrm{~min}$ and a limit of $10 \mathrm{mg} /$ hour. No other pro re nata (PRN) analgesia was provided.

\section{Patient and public involvement}

Patients and/or the public were not involved in the design, or conduct, or reporting, or dissemination plans of this research.

\section{Data collection}

Demographic and intraoperative characteristics including age, ASA classification, height, weight, BMI and surgical duration were recorded. The following medication data points were recorded: cumulative morphine dose in the first 24 hours, cumulative morphine dose administered from 0 to 1,1 to 6,6 to 12,12 to 18 , and 18 to 24 hours after surgery, and the time from the end of surgery to the first need for morphine analgesia. Visceral and incisional pain intensity scores (at rest and on movement) were recorded at $0.5,1,2,6,12,18$, and 24 hours after surgery as NRS scores ranging from 0 to 10 . Adverse reactions during and after surgery were recorded, including nausea and vomiting, itchy skin, puncture site infection, abdominal organ injury, local anaesthetic toxicity, hypokinesia of the lower extremity and paresthesia.

\section{Statistical analysis}

All data were analysed with SPSS V.22.0 statistical software. The Shapiro-Wilk test was used to evaluate normal distribution. Measured data with normal distribution were expressed as mean $\pm \mathrm{SD}$; non-normally distributed data were expressed as median (IQR). Frequencies were used for categorical variables. ASA classification and incidence of adverse events were analysed with the $\mathrm{X}^{2}$ test. Age, height, weight, BMI and 24-hour cumulative morphine dose were analysed with Student's t-test. Surgical duration and morphine dose in the intervals were analysed with the Mann-Whitney U test. Kaplan-Meier survival curves were used to plot the elapsed time between completion of surgery and the first administration of rescue analgesia; the difference between groups was compared with the log-rank test. NRS scores were analysed with repeatedmeasures analysis of variance. Values of $p<0.05$ were considered statistically significant.

\section{Sample size calculation}

The power analysis was based on the primary endpoint of cumulative morphine dose in the first 24 hours after surgery in preliminary study, and performed with the Power Sample Size (PASS V.11.0) software program (NCSS, Kaysville, Utah, USA). In the preliminary study, 10 patients were assigned to a TQL or OSTAP group $(n=5)$, and the cumulative morphine dose in 24 hours was compared between groups $(15.0 \pm 14.2$ vs $28.2 \pm 6.1 \mathrm{mg}$ ). We assumed a type I error of 0.05 and a power of 0.90, and the Student's t-test was used. A sample size of 21 patients per group was needed to determine statistically significant differences between groups. Considering the loss potential and errors, we decided to include 30 patients in each group for this trial.

\section{RESULTS}

\section{General information}

Seventy-two female patients for elective laparoscopic hysterectomy between September 2018 and March 2019 were considered eligible; of these 60 patients were randomly assigned and included in the analysis (figure 2). There was no statistically significant 


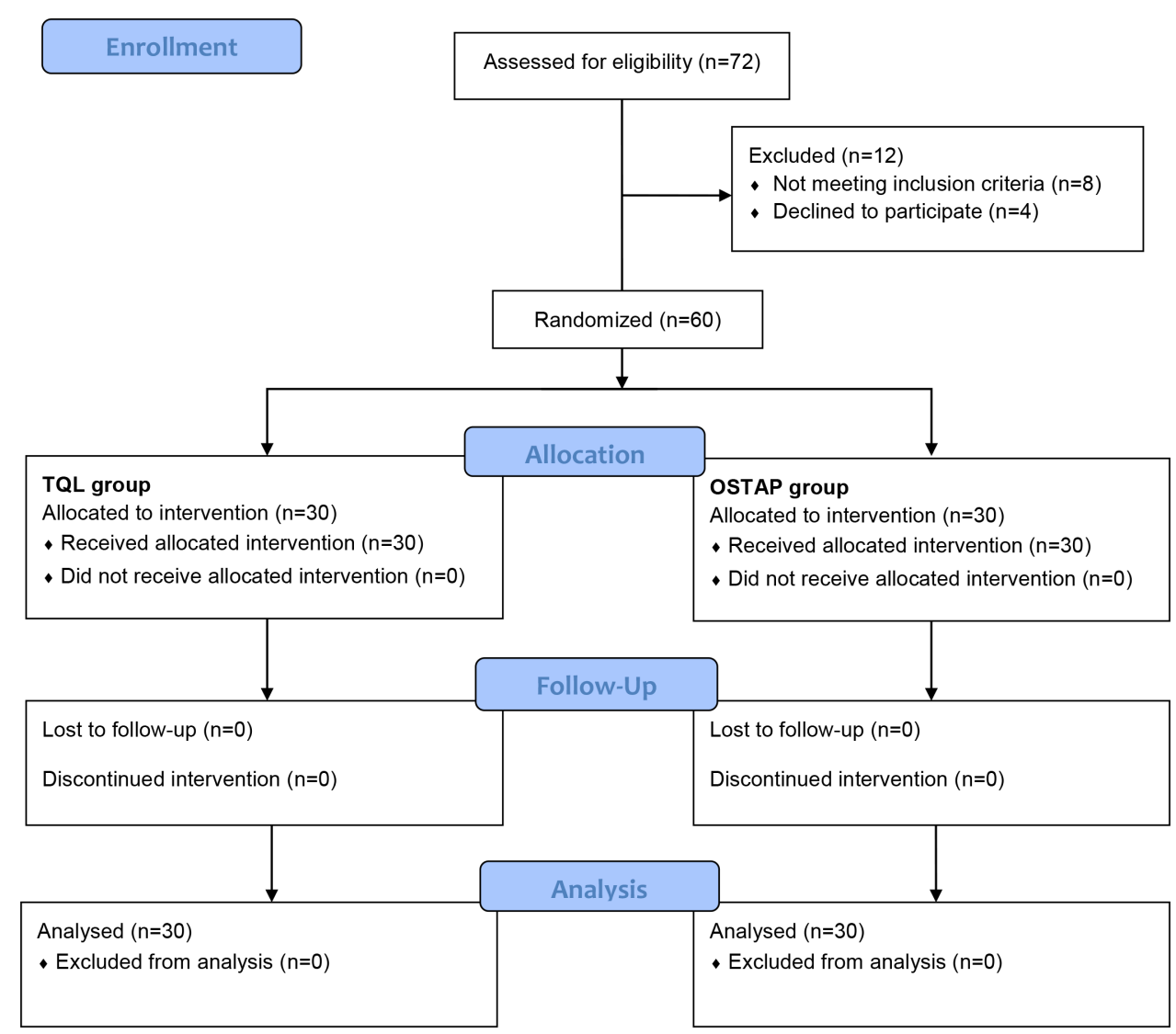

Figure 2 CONSORT flow diagram. CONSORT, CONSolidated Standards Of Reporting Trials; OSTAP, oblique subcostal transversus abdominis plane; TQL, transmuscular quadratus lumborum block.

difference between groups in ASA classification, age, height, weight, BMI or surgical duration (table 1).

\section{Morphine consumption and the time of first need for morphine} The cumulative morphine dose in the first 24 hours after surgery was significantly lower in the TQL group than in the OSTAP group $(p=0.010)$. The morphine doses administered in the intervals from 6 to 12,12 to 18 , and 18 to 24 hours were significantly lower in TQL group than in the OSTAP group $(p=0.003, p=0.020$, $\mathrm{p}=0.028$, respectively), but no significant difference in the intervals from 0 to 1,1 to 6 hours $(\mathrm{p}=0.321$ and $\mathrm{p}=0.845)($ table 2$)$.
The median time in hours between completion of surgery and the first administration of rescue analgesia was significantly longer in the TQL group than in the OSTAP group $(6.0 ; 95 \%$ CI 5.5 to 6.5 vs $4.0 ; 95 \%$ CI 3.1 to 4.9 hours, $\mathrm{p}=0.027$, computed using a log-rank test) (figure 3).

\section{Pain intensity scores}

The NRS scores for postoperative visceral pain intensity at rest and on movement in TQL group were significantly lower than that in the OSTAP group $(p=0.007$ and $\mathrm{p}<0.001$, respectively) (figure $4 \mathrm{~A}, \mathrm{~B}$ ). The NRS scores for postoperative incisional pain intensity at

\begin{tabular}{llll}
\hline \multicolumn{1}{l}{ Table 1 Demographic and intraoperative characteristics } & & \\
\hline Characteristic & OSTAP group, $\mathbf{n}=\mathbf{3 0}$ & TQL group, $\mathbf{n = 3 0}$ & P value \\
\hline ASA classification (I/II) & $19(63.3) / 11(36.7)$ & $18(60) / 12(40)$ & 0.791 \\
Age, years & $50.6 \pm 6.2$ & $52.6 \pm 7.5$ & 0.256 \\
Height, $\mathrm{cm}$ & $158.5 \pm 5.6$ & $158.8 \pm 5.2$ & 0.867 \\
Weight, $\mathrm{kg}$ & $59.2 \pm 8.9$ & $59.8 \pm 8.4$ & 0.807 \\
BMI, $\mathrm{kg} / \mathrm{m}^{2}$ & $23.5 \pm 3.0$ & $23.6 \pm 2.6$ & 0.869 \\
Surgical duration, $\min$ & $120.0(93.8-150.0)$ & $120.0(90.0-122.5)$ & 0.195 \\
\hline
\end{tabular}

Measured data with normal distribution were expressed as mean \pm SD. Non-normal distributed data are expressed as median (IQR). Qualitative data were expressed as $n(\%)$.

ASA, American Society of Anesthesiologists; BMI, body mass index; OSTAP, oblique subcostal transversus abdominis plane; TQL, transmuscular quadratus lumborum. 


\begin{tabular}{|c|c|c|c|}
\hline $\begin{array}{l}\text { Morphine } \\
\text { consumption }\end{array}$ & $\begin{array}{l}\text { OSTAP group, } \\
\mathrm{n}=30\end{array}$ & $\begin{array}{l}\text { TQL group, } \\
n=30\end{array}$ & P value \\
\hline \multicolumn{4}{|c|}{ Interval morphine consumption, mg } \\
\hline 0-1 hour & $0.0(0.0-0.0)$ & $0.0(0.0-0.0)$ & 0.321 \\
\hline 1-6hours & $3.8(1.5-9.4)$ & $4.5(0.0-9.8)$ & 0.845 \\
\hline $6-12$ hours & $14.2(6.0-15.0)$ & $7.5(3.0-12.0)$ & 0.003 \\
\hline 12-18hours & $3.0(1.1-9.8)$ & $0.8(0.0-6.0)$ & 0.02 \\
\hline 18-24 hours & $0.0(0.0-3.0)$ & $0.0(0.0-0.0)$ & 0.028 \\
\hline \multicolumn{4}{|c|}{ Cumulative morphine consumption, mg } \\
\hline 24 hours & $26.1 \pm 13.3$ & $17.2 \pm 12.5$ & 0.01 \\
\hline
\end{tabular}

Measured data with normal distribution were expressed as mean \pm SD. Non-normal distributed data are expressed as median (IQR).

OSTAP, oblique subcostal transversus abdominis plane; TQL, transmuscular quadratus lumborum.

rest and on movement were not significantly different between groups $(\mathrm{p}=0.507$ and $\mathrm{p}=0.640$, respectively) (figure 4C,D).

\section{Adverse events}

There were no significant differences between two groups in the incidence of postoperative nausea and vomiting (7 patients in the TQL group, 14 patients in the OSTAP group, $\mathrm{p}=0.058$ ). No other adverse events were observed in either group, including puncture site infection, abdominal organ injury, local anaesthetic toxicity, lower extremity muscle weakness or skin itching.

\section{DISCUSSION}

In this study, we compared the postoperative analgesic effect of the TQL block with OSTAP block in patients undergoing laparoscopic hysterectomy. Results showed that patients who received a TQL block had significantly lower morphine consumption and lower visceral pain intensity

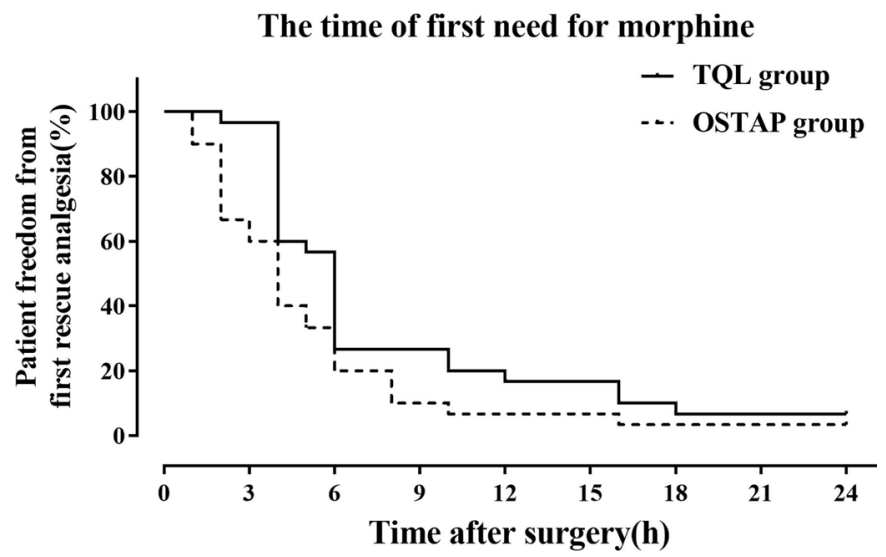

Figure 3 Time of first need for morphine demonstrated by the Kaplan-Meier survival curves $(6.0 ; 95 \% \mathrm{Cl} 5.5$ to 6.5 vs 4.0; $95 \% \mathrm{Cl} 3.1$ to 4.9 hours, $\mathrm{p}=0.027$, computed using a logrank test). OSTAP, oblique subcostal transversus abdominis plane; TQL, transmuscular quadratus lumborum block. scores over 24 hours after surgery, which demonstrated that the TQL block had longer duration and more sufficient strength of analgesia when compared with OSTAP block.

Blanco et alfirst described that the effect of lateral QL block was similar to that of the TAP block. Meanwhile, Blanco et al moved the point of injection to the posterior wall of the $\mathrm{QL}$ (posterior QL block), considering the posterior QL block results in a more extensive sensory-level analgesic. ${ }^{13}$ Dam et al injected local anaesthetic into the interfascial plane between the QL and psoas major (TQL block), ${ }^{16}$ and also studies confirmed that TQL block provided more effective postoperative analgesic effect compared with posterior QL block. ${ }^{17}{ }^{18}$ Based on this background, the TQL block was chosen in this study. Case reports and randomised controlled clinical trials still did not reach a consensus on the block range generated by different approaches of TQL block. A cadaver study compared the different approaches of QL block, showing that the analgesic range may be from T10 to L 4 by TQL block. ${ }^{19}$ Also, there was a case series study that demonstrated that the TQL block at L2 level generated a block range from $\mathrm{T} 8$ to $\mathrm{L} 1{ }^{20} \mathrm{Lu}$ et al reported that the TQL block primarily affected dermatomes T9-L1, while the maximum cephalad dermatome level reached was T7, and caudally was L2. ${ }^{21}$ These studies revealed that the TQL block is suitable for postoperative analgesia after abdominal surgery, especially for lower abdominal surgery.

In this study, a $10 \mathrm{~cm}$ needle retracted and reinserted into the plane along the oblique subcostal line four to five times to accomplish the OSTAP block as Chen $e t a l^{5}$ described, rather than the $15-20 \mathrm{~cm}$ needle used by Hebbard et al. ${ }^{5}$ In addition, the effective hydrodissection allowed a less volume of local anaesthetic than that used by Hebbard et $a l .{ }^{515}$ Several studies confirmed that the OSTAP block could provide postoperative analgesia for abdominal surgery. Lee et al demonstrated that an OSTAP block blocked four segments on the anterior abdominal wall with the most cephalad T8 using a $20 \mathrm{~mL}$ volume. ${ }^{22}$ Chen et al demonstrated that OSTAP block produced a cutaneous sensory blockade with a consistent dermatomal distribution from T6 to L1 $1{ }^{15}$ Meanwhile, the OSTAP block reduced tramadol requirements and Visual Analogue Scale scores within 24 hours after laparoscopic hysterectomy in a randomised controlled trial. ${ }^{23}$ However, a study also reported that the analgesic effect of subcostal TAP was limited in laparoscopic cholecystectomy, because patients complained more of visceral pain than of incisional pain. ${ }^{24}$ There were no significant differences in incisional pain scores between the groups. However, the visceral pain scores were significantly higher in the OSTAP group than in the TQL group. Meanwhile, the morphine consumption in the TQL group was significantly reduced 6 hours after surgery. Therefore, it is considered that the OSTAP block relieved incisional pain due to the local anaesthetic infiltrate into the anterior abdominal wall, but has limited effects on relieving visceral pain.

The TQL block and OSTAP block are trunk nerve block techniques, however the TQL block produced longer analgesia duration and relieved both incisional and visceral 
A NRS score for visceral pain at rest

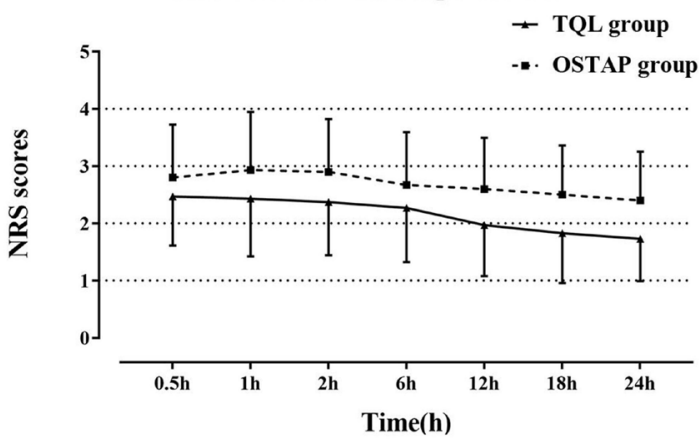

C NRS score for incisional pain at rest

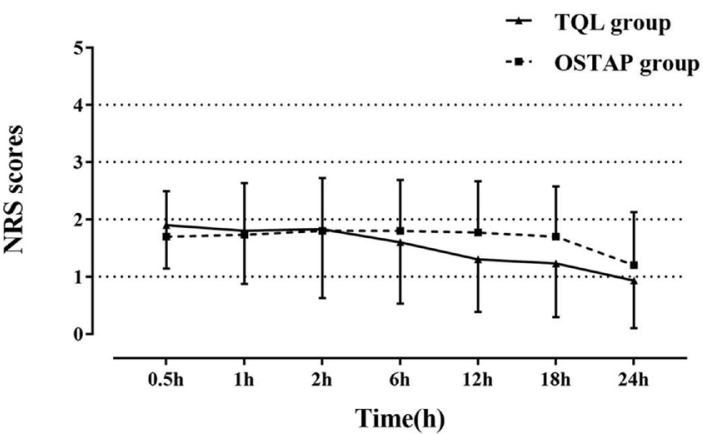

B NRS score for visceral pain on movement

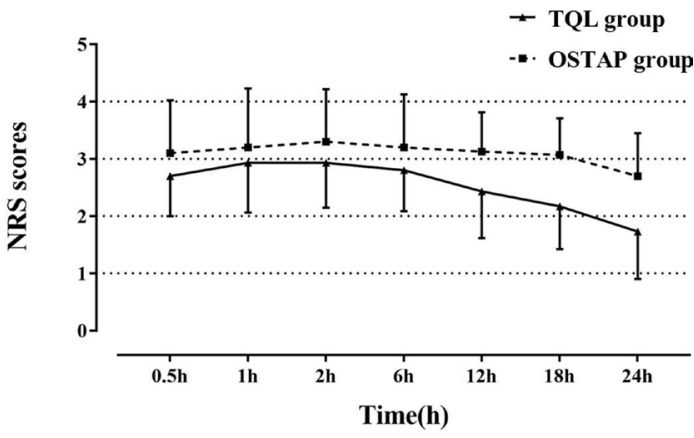

D NRS score for incisional pain on movement - TQL group

-. OSTAP group

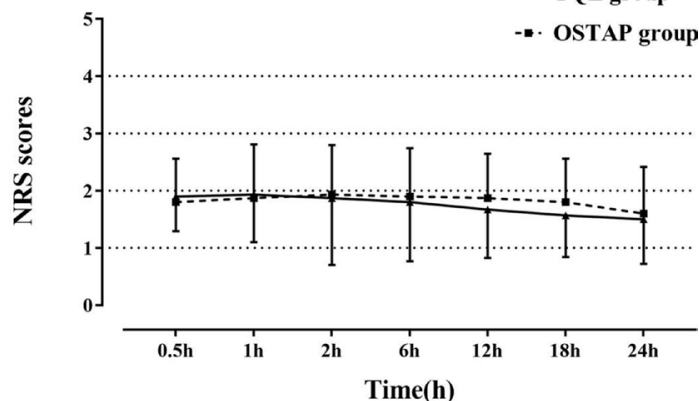

Figure 4 NRS scores for postoperative visceral and incisional pain intensity (at rest and on movement). (A) NRS score for visceral pain at rest; (B) NRS score for visceral pain on movement; (C) NRS score for incisional pain at rest; (D) NRS score for incisional pain on movement. Data were expressed as mean \pm SD. NRS, Numerical Rating Scale; OSTAP, oblique subcostal transversus abdominis plane; TQL, transmuscular quadratus lumborum.

pain in this study. The mechanism of the TQL block has not been clarified. Most researchers believe that the local anaesthetics spread to the thoracic paravertebral space through the thoracolumbar fascia, producing effects similar to a paravertebral nerve block. Carline et al found that dye injected in the anterior QL in cadavers spread to the psoas major and QL muscles, and surrounded the roots of the first to third lumbar nerves. ${ }^{19}$ Dam et al found that after TQL block in cadavers, the dye spread into the thoracic paravertebral space and the intercostal spaces to surround the somatic nerves and thoracic sympathetic trunk. ${ }^{25}$ In addition, there is reticular distribution of sympathetic nerve fibres and a rich neural network in the fascial structures, ${ }^{26}$ it was confirmed that sympathetic nerves play an important role in the occurrence and progression of pain. ${ }^{27}$ The TQL block might produce analgesic effects by blocking sympathetic nerve fibres in the thoracolumbar fascia.

No puncture site infection, abdominal organ injury, local anaesthetic toxicity, weakness of lower limbs or skin itching occurred in this study, indicating that both the TQL block and OSTAP block were safe. In both the TQL group and OSTAP group, different degrees of nausea and vomiting occurred, a finding that might be attributed to surgery, general anaesthesia, postoperative use of morphine and other factors. There was no significant difference in the incidence of nausea and vomiting between the groups, the $\mathrm{p}$ value was close to 0.05 .

This study had several limitations. (1) Although patients received standardised education to distinguish between the incisional and visceral pain, a small proportion of patients could not distinguish the two types of pain clearly, but were able to give NRS scores for visceral and incisional pain, respectively, with the guidance of the assessors. (2) Some of the secondary outcomes were not assessed but were initially included in the registration form. The Bruggrmann Comfort Scale score is evaluated in patients with deep breathing or coughing, similar to NRS scores assessed both at rest and on movement in this study. The investigator could not correctly record the time when the patients got out of bed within 24 hours after surgery due to an unforeseen circumstance that some patients refused early postoperative activity, affected by the traditional Chinese concepts. Ramsay Sedation Scale score (RSS) is widely used to evaluate the degree of sedation. In the preliminary study, patients did not consume much morphine in two groups, and were all awake and cooperative after the operation, so we did not observe RSS in this study. Meanwhile, we added the observation time point of 12 hours for evaluating NRS pain score in this study, which had not been prespecified in the trial registry. (3) Because of the limited sample size of this study, the $\mathrm{p}$ value was close to 0.05 when the incidence of nausea and vomiting was compared between groups. A larger sample size is needed to confirm this finding. (4) We excluded the patients with BMI $\geq 28 \mathrm{~kg} / \mathrm{m}^{2}$ or $\leq 18 \mathrm{~kg} / \mathrm{m}^{2}$, which limits the generalisability of the results. (5) Since the local anaesthetic flows back from the needle path of each insertion when performing the OSTAP block, a slight difference in the volume of the local anaesthetic between the two groups may bring the risk of bias. (6) Inhalation combined with venous anaesthesia was used in this study, which was 
beneficial in reducing anaesthetic-related adverse reactions. However, one more variable may lead to biased result.

\section{CONCLUSIONS}

In this study, the TQL block provided better visceral pain relief with longer duration of analgesia and reduced postoperative morphine consumption, compared with the OSTAP block. As a new developing regional anaesthesia technique, the TQL block may be a component of multimodal analgesia for postoperative pain relief following laparoscopic hysterectomy and other laparoscopic surgeries.

Acknowledgements We would like to thank Rebecca Tollefson, from Liwen Bianji, Edanz Editing China (www.liwenbianji.cn/ac), for editing the English text of a draft of this manuscript.

Contributors LH and LZ are joint first authors. KS obtained funding. KS and QW designed the study. XZ and LP collected the data. YZ was involved in data cleaning. $\mathrm{JZ}$ and $\mathrm{YZ}$ analysed the data. LH and LZ drafted the manuscript. KS and QW contributed to the interpretation of the results and critical revision of the manuscript for important intellectual content and approved the final version of the manuscript. All authors have read and approved the final manuscript. KS is the study guarantor.

Funding This work was supported by the National Natural Science Foundation of China (grant no. 81900231).

Competing interests None declared.

Patient and public involvement Patients and/or the public were not involved in the design, or conduct, or reporting, or dissemination plans of this research.

Patient consent for publication Not required.

Ethics approval This study was approved by the Ethical Committee of The First Affiliated Hospital of Wenzhou Medical University, Wenzhou, China (No. 108, 1 August 2018).

Provenance and peer review Not commissioned; externally peer reviewed.

Data availability statement Data are available upon reasonable request. Data are available upon reasonable request. The technical appendix, statistical code and data set are available from the corresponding author.

Open access This is an open access article distributed in accordance with the Creative Commons Attribution Non Commercial (CC BY-NC 4.0) license, which permits others to distribute, remix, adapt, build upon this work non-commercially, and license their derivative works on different terms, provided the original work is properly cited, appropriate credit is given, any changes made indicated, and the use is non-commercial. See: http://creativecommons.org/licenses/by-nc/4.0/.

ORCID iD

Kejian Shi http://orcid.org/0000-0003-1396-7424

\section{REFERENCES}

1 Gerbershagen HJ, Aduckathil S, van Wijck AJM, et al. Pain intensity on the first day after surgery: a prospective cohort study comparing 179 surgical procedures. Anesthesiology 2013;118:934-44.

2 Choi JB, Kang K, Song MK, et al. Pain characteristics after total laparoscopic hysterectomy. Int J Med Sci 2016;13:562-8.

3 Frassanito L, Zanfini BA, Catarci S, et al. Erector spinae plane block for postoperative analgesia after total laparoscopic hysterectomy: case series and review of the literature. Eur Rev Med Pharmacol Sci 2020;24:3892-7.

4 Gupta C, Valecha UK, Singh SP, et al. Systemic lidocaine versus ultrasound-guided transversus abdominis plane block for postoperative analgesia: a comparative randomised study in bariatric surgical patients. Indian J Anaesth 2020;64:31-6.

5 Hebbard PD, Barrington MJ, Vasey C. Ultrasound-Guided continuous oblique subcostal transversus abdominis plane blockade: description of anatomy and clinical technique. Reg Anesth Pain Med 2010;35:436-41.
6 Shin H-J, Oh A-Y, Baik J-S, et al. Ultrasound-Guided oblique subcostal transversus abdominis plane block for analgesia after laparoscopic cholecystectomy: a randomized, controlled, observerblinded study. Minerva Anestesiol 2014;80:185-93.

7 Basaran B, Basaran A, Kozanhan B, et al. Analgesia and respiratory function after laparoscopic cholecystectomy in patients receiving ultrasound-guided bilateral oblique subcostal transversus abdominis plane block: a randomized double-blind study. Med Sci Monit 2015;21:1304-12.

8 Aygun H, Kavrut Ozturk N, Pamukcu AS, et al. Comparison of ultrasound guided erector spinae plane block and quadratus lumborum block for postoperative analgesia in laparoscopic cholecystectomy patients; a prospective randomized study. J Clin Anesth 2020;62:109696.

9 Aditianingsih D, Pryambodho P, Anasy N. A randomized controlled trial on analgesic effect of repeated quadratus lumborum block versus continuous epidural analgesia following laparoscopic nephrectomy. BMC Anesthesiol 2019;19:221.

10 Ökmen K, Metin Ökmen B, Topal S. Ultrasound-guided posterior quadratus lumborum block for postoperative pain after laparoscopic cholecystectomy: a randomized controlled double blind study. J Clin Anesth 2018;49:112-7.

11 Ishio J, Komasawa N, Kido H, et al. Evaluation of ultrasound-guided posterior quadratus lumborum block for postoperative analgesia after laparoscopic gynecologic surgery. J Clin Anesth 2017;41:1-4.

12 Blanco R, Ansari T, Riad W, et al. Quadratus lumborum block versus Transversus abdominis plane block for postoperative pain after cesarean delivery: a randomized controlled trial. Reg Anesth Pain Med 2016;41:757-62.

13 Blanco R, Ansari T, Girgis E. Quadratus lumborum block for postoperative pain after caesarean section: a randomised controlled trial. Eur J Anaesthesiol 2015;32:812-8.

14 Ueshima H, Otake H, Lin J-A. Ultrasound-guided quadratus lumborum block: an updated review of anatomy and techniques. Biomed Res Int 2017;2017:2752876

15 Chen Y, Shi K, Xia Y, et al. Sensory assessment and regression rate of bilateral oblique subcostal Transversus abdominis plane block in volunteers. Reg Anesth Pain Med 2018;43:174-9.

16 Dam M, Hansen CK, Børglum J, et al. A transverse oblique approach to the transmuscular quadratus lumborum block. Anaesthesia 2016;71:603-4.

17 Bagbanci O, Kursad $\mathrm{H}$, Yayik AM, et al. Comparison of types 2 and 3 quadratus lumborum muscle blocks : Open inguinal hernia surgery in patients with spinal anesthesia. Anaesthesist 2020;69:397-403.

18 Ahmed A, Fawzy M, Nasr MAR, et al. Ultrasound-guided quadratus lumborum block for postoperative pain control in patients undergoing unilateral inguinal hernia repair, a comparative study between two approaches. BMC Anesthesiol 2019;19:184.

19 Carline L, McLeod GA, Lamb C. A cadaver study comparing spread of dye and nerve involvement after three different quadratus lumborum blocks. Br J Anaesth 2016;117:387-94.

20 Kadam VR, Howell S. Ultrasound-guided continuous transmuscular quadratus lumborum block- L4 or L2 level catheter insertion for analgesia in open abdominal surgery: case series. Indian $J$ Anaesth 2018:62:555-7.

21 Lu Y, Zhang J, Xu X, et al. Sensory assessment and block duration of transmuscular quadratus lumborum block at $L 2$ versus $L 4$ in volunteers: a randomized controlled trial. Minerva Anestesiol 2019;85:1273-80.

22 Lee THW, Barrington MJ, Tran TMN, et al. Comparison of extent of sensory block following posterior and subcostal approaches to ultrasound-guided transversus abdominis plane block. Anaesth Intensive Care 2010;38:452-60.

23 Korkmaz Toker M, Altiparmak B, Uysal Ali İhsan, et al. The analgesic efficacy of oblique subcostal transversus abdominis plane block after laparoscopic hysterectomy: a randomized, controlled, observerblinded study. Medicine 2019;98:e13994.

24 Houben AM, Moreau A-SJ, Detry OM, et al. Bilateral subcostal transversus abdominis plane block does not improve the postoperative analgesia provided by multimodal analgesia after laparoscopic cholecystectomy: a randomised placebo-controlled trial. Eur J Anaesthesiol 2019;36:772-7.

25 Dam M, Moriggl B, Hansen CK, et al. The pathway of Injectate spread with the transmuscular quadratus lumborum block: a cadaver study. Anesth Analg 2017;125:303-12.

26 Benetazzo L, Bizzego A, De Caro R, et al. 3D reconstruction of the crural and thoracolumbar fasciae. Surg Radiol Anat 2011;33:855-62.

27 Baron R, Jänig W. [Pain syndromes with causal participation of the sympathetic nervous system]. Anaesthesist 1998;47:4-23. 